\title{
Modelagem para a dinâmica populacional do Aedes aegypti via simetrias de Lie
}

\author{
Felipo Bacani \\ Departamento de Matemática Aplicada, IMECC - UNICAMP \\ Cidade Universitária Zeferino Vaz, Barão Geraldo, 13083-970, Campinas, SP \\ E-mail: bacani@ime.unicamp.br
}

Igor L. Freire Norberto A. Maidana

Centro de Matemática, Computação e Cognição, Universidade Federal do ABC - UFABC

Rua Santa Adélia, 166, Bairro Bangu, 09.210-170, Santo André, SP

E-mail: igor.freire@ufabc.edu.br_norberto.maidana@ufabc.edu.br

\author{
Mariano Torrisi \\ Dipartimento di Matematica e Informatica, Università Degli Studi di Catania, \\ Viale Andrea Doria, 6, 95125 Catania, Itália \\ E-mail: torrisi@dmi.unict.it
}

\begin{abstract}
Resumo: Neste trabalho são estudados modelos matemáticos que descrevem a dinâmica vital e espacial do mosquito Aedes aegypti, principal transmissor da dengue, e sua relação com a população humana. Os modelos analisados têm a dinâmica espacial (difusão e transporte) não-linear, isto é, dependentes da densidade de mosquitos, e a dinâmica vital malthusiana, isto é, sem competição inter-específica. O estudo analítico dos modelos foi feito através da técnica de simetrias de Lie, que permite obter informações sobre o sistema de equações diferenciais em questão, como soluções especiais e trocas de coordenadas que simplifiquem o problema. Esta técnica permitiu encontrar soluções do tipo onda viajante dos modelos estudados, além de soluções adicionais provenientes de simetrias não triviais do modelo.
\end{abstract}

Palavras-chave: Aedes aegypti, Modelagem matemática, Simetrias de Lie

\section{Introdução}

Modelos matemáticos permitem obter informação para uma melhor compreensão de um fenômeno, mas também fornecem subsídios quantitativos para avaliar diferentes aspectos a serem estudados. No caso do estudo de doenças, possíveis estratégias de controle podem ser avaliadas com base nos resultados da modelagem de cada situação específica. Especificamente para a dengue, atualmente não há perspectiva de uma vacina efetiva e de produção barata, portanto toda política pública para controle da doença deve necessariamente incluir estratégias que visem o controle da população do mosquito transmissor. Nesse sentido, a utilização de modelos matemáticos como ferramenta para compreender e prever a dinâmica da transmissão da doença, isto é, a dinâmica entre o mosquito e o ser humano, ganha ainda mais relevância.

Neste trabalho pretendemos aplicar técnicas de simetrias de Lie no estudo de modelos biomatemáticos. Tal técnica tem suas aplicações mais notórias em equações que descrevem fenômenos da física e matemática aplicada. Contudo a aplicação de simetria de Lie à modelos de fenômenos biológicos tem sido pouco frequente, mesmo que grande parte dos modelos em biomatemática sejam descritos por equações diferenciais. Dessa forma, tal ferramenta ganha importância, na medida que fornece informações analíticas sobre o modelo em questão, como trocas de variáveis que simplifiquem o problema ou soluções para condições específicas dos parâmetros do modelo. 
Um dos primeiros trabalhos a aplicarem a técnica de simetrias de Lie a modelos envolvendo populações foi [6], trabalho no qual modelos de colônias de bactérias foram analisados.

A utilização da técnica de simetrias à problemas da epidemiologia modelados por equações diferenciais parciais foi feita recentemente pelo por dois de nós, em $[3,4]$, onde modelos da dinâmica populacional do Aedes aegypti foram analisados via simetrias de Lie, incluindo a construção de soluções exatas para tais modelos.

\section{Motivação}

A motivação principal deste trabalho são os trabalhos [1,2], relacionados à modelagem do Aedes aegypti, e no novo modelo para a dinâmica populacional do mosquito, introduzido pelo proponente e seu colaborador [3,4]. Os modelos considerados aqui trabalham com duas fases do mosquito, a fase aquática e a fase alada, e permitem determinar distintos tipos de estratégias de controle tanto da dinâmica local, como da dispersão espacial do mosquito.

O modelo introduzido nas referências $[1,5]$, que inspiraram os trabalhos [3,4], é introduzido a seguir. Considere o seguinte sistema:

$$
\left\{\begin{array}{l}
\bar{u}_{\hat{t}}=D \bar{u}_{\hat{x} \hat{x}}-\bar{\nu} u_{\hat{x}}+\bar{\gamma} \bar{v}\left(1-\frac{\bar{u}}{\bar{k}_{1}}\right)-\bar{\mu}_{1} \bar{u} \\
\bar{v}_{\hat{t}}=\bar{r} \bar{u}\left(1-\frac{\bar{v}}{\bar{k}_{2}}\right)-\left(\bar{\mu}_{1}+\bar{\gamma}\right) \bar{v}
\end{array}\right.
$$

onde $\bar{u} \equiv \bar{u}(\hat{x}, \hat{t})$ e $\bar{v} \equiv \bar{v}(\hat{x}, \hat{t})$ são respectivamente as populações aquáticas e aladas do mosquito em função da posição $\hat{x}$ e do instante $\hat{t}$.

O crescimento de $\bar{u}$ (isto é, a taxa específica de maturação da fase aquática em alada) é dada pelo terceiro termo do lado direito da primeira equação do sistema (1). O crescimento de $\bar{u}$ é proporcional à quantidade de ovos $\bar{v}$ e à constante $\bar{\gamma}$ (a taxa específica de maturação da fase aquática em mosquitos fêmeas), com saturação dada pela capacidade suporte $\bar{k}_{1}$ (quantidade de recursos atingíveis, i.e. sangue humano disponível). De forma totalmente análoga, o aumento de $\bar{v}$ (isto é, a taxa de oviposição de ovos por fêmeas de mosquitos), representado pelo primeiro termo do lado direito do sistema (1) acima, é proporcional à quantidade de mosquitos $\bar{u}$ e à taxa de crescimento constante $\bar{r}$, e tem saturação dada pela constante $\bar{k}_{2}$ (quantidade de criadouros disponíveis).

Considerando a dinâmica espacial do sistema (1), é considerada a dispersão do Aedes aegypti como resultado de voos aleatórios e locais, macroscopicamente representado por um processo difusivo com coeficiente constante $\bar{D}$, acoplado à advecção (transporte) do vento dada por um fluxo constante $\bar{\nu}[8]$. A advecção constante pode ser justificada como 'viés' (bias) no transporte de grandes quantidades de mosquitos (portanto um fator importante no modelo), causada por uma corrente de vento que em geral tem por características ter intensidade, duração e de direção geográfica relevantes. Flutuações de correntes de vento de menores amplitudes, portanto de menor relevância no transporte do mosquito, são considerados como parte do efeito difusivo do modelo.

De forma a representar apropriadamente as escalas do modelo, o sistema (1) é adimensionalizado, para $\bar{u}=u / \bar{k}_{1}, \bar{v}=v / \bar{k}_{2}, t=\bar{t} \cdot \bar{r}$ e $x=\bar{x} \cdot \sqrt{\bar{D}} \bar{r}^{-1}$, e os cinco parâmetros adimensionais

$$
\mu_{1}=\frac{\bar{\mu}_{1}}{\bar{r}}, \quad \mu_{2}=\frac{\bar{\mu}_{2}}{\bar{r}}, \quad \gamma=\frac{\bar{\gamma}}{\bar{r}}, \quad \nu=\frac{\bar{\nu}}{\sqrt{\bar{r} \bar{D}}}, \quad k=\frac{\bar{k}_{1}}{\bar{k}_{2}},
$$

originando o sistema abaixo:

$$
\left\{\begin{array}{l}
u_{t}=u_{x x}-\nu u_{x}+\frac{\gamma}{k} v(1-u)-\mu_{1} u \\
v_{t}=k u(1-v)-\left(\mu_{2}+\gamma\right) v
\end{array}\right.
$$




\section{Modelos Estudados}

Inspirados no modelo dado pelo sistema (2), I. L. Freire e M. Torrisi [3], introduziram nãolinearidades nos efeitos de difusão e transporte ao sistema (2), e realizar as modificações a seguir:

1. Retira-se a autorregulação da espécie, isto é, o termo logístico, dado por o termo misto " $u v$ " da primeira equação e agrupa-se " $(\gamma / k) u$ ", de forma a retirar a saturação de crescimento dos mosquitos. O termo $\left(\frac{\gamma}{k}-\mu_{1}\right) u$ em função da capacidade suporte $k$ considera uma taxa de natalidade e mortalidade malthusiana proporcional aos parâmetros de interesse na equação não linear, isto é, natalidade dependente da taxa de eclosão e capacidade do meio, e mortalidade $\mu_{1}$ que pode ser aumentada por controle da população de mosquitos na fase alada;

2. Similarmente à modificação anterior, retira-se a autoregulação da espécie, dada por o termo em " $u v$ " da segunda equação e agrupando " $k v$ " (retirando assim a saturação na fase aquática por falta de criadouros).

3. Considera-se a difusão das populações e advecção dependentes da densidade dos mesmos, características das populações de insetos [8], que não foram consideradas em (1) e (2) para simplificação do modelo.

Resultando assim em um modelo (3), mais rico em simetrias devido à não-linearidade na difusão e transporte.

$$
\left\{\begin{array}{l}
u_{t}=\left(u^{p} u_{x}\right)_{x}-2 \nu u^{q} u_{x}+\frac{\gamma}{k} v+\left(\frac{\gamma}{k}-\mu_{1}\right) u \\
v_{t}=k u+\left(k-\mu_{2}-\gamma\right) v
\end{array}\right.
$$

A dinâmica vital do modelo (3) aproxima a de (2), no sentido que não há saturação de crescimento, portanto é capaz de descrever apenas um ciclo de vida do mosquito. Situações biológicas onde apenas um ciclo de vida é relevante à dinâmica dos mosquitos podem ser locais onde infestações ocorrem muito rapidamente.

Por outro lado, a dinâmica espacial de (3) engloba a de (2) (onde a difusão e o transporte são consideradas constantes por simplificação), ao descrever os efeitos de difusão e transporte através de termos não-lineares.

Durante a classificação de simetrias de Lie do sistema (3) obtida em [3], o caso $\nu=0$ surgiu como o caso não-linear admitindo o maior número de simetrias de Lie. Biologicamente tal modelo significa que os efeitos devidos a correntes de vento são desprezíveis. Isso pode ocorrer, por exemplo, quando a região onde há infestação possui barreiras naturais que não permitem correntes de vento agindo naquela região.

Tal sistema, com $\nu=0$, foi melhor analisado em [4], onde as técnicas de simetrias foram utilizadas para se encontrar soluções explícitas do sistema. Em particular, o uso de simetrias possibilitou a transformação do sistema de equações diferenciais original num sistema de EDOs de primeira ordem. Para tal sistema foram obtidas várias soluções, ao passo que uma análise qualitativa também foi considerada. Neste caso foi obtida a condição de existência da infestação, que levou a resultados similares aos obtidos em [2].

No estudo da dinâmica do $A$. aegypti, é importante determinar a eficiência de diferentes tipos de controles da população de mosquitos. Mas uma vez que o interesse principal da dengue é a transmissão da doença, é necessário incluir o ser humano na modelagem.

Para a dinâmica do mosquito é considerado o modelo proposto em (2), ao qual se acopla a população de humanos. Consideram-se as subpopulações de mosquitos suscetíveis e infectados na fase alada (respectivamente $u$ e $v$ ), assim como a subpopulações de humanos suscetíveis, infectados e recuperados $(h, i$ e $r$ ). Não é considerada a subpopulação de recuperados nos 
mosquitos, por que uma vez infetados, estes ficam assim durante toda a sua vida. A dinâmica é dada pelo sistema abaixo:

$$
\left\{\begin{aligned}
u_{t} & =u_{x x}-\nu u_{x}+\frac{\gamma}{k} v\left(1-\frac{m}{k_{1}}\right)-\mu_{1} u-\beta_{1} u i \\
w_{t} & =w_{x x}-\nu w_{x}-\mu_{1} w+\beta_{1} u i \\
v_{t} & =k(1-v) m-\left(\mu_{2}+\gamma\right) v \\
h_{t} & =\mu_{h} N-\mu_{h} h-\beta_{2} h w \\
i_{t} & =\beta_{2} h w-\sigma i-\mu_{h} i \\
r_{t} & =\sigma i-\mu_{h} r
\end{aligned}\right.
$$

onde $u$ é a subpopulação de mosquitos suscetíveis, $w$ a subpopulação de mosquitos infectados, $m=(u+w)$ é a população total de mosquitos e $v$ corresponde à população na fase aquática. A população humana, denotada por $N$ é dividida em três subpopulações, suscetíveis, infectados e recuperados. Os novos parâmetros envolvidos são as taxas de contágio humano-vetor $\beta_{1}$, e vetor-humano, $\beta_{2}$. Na população de humanos é considerada a mortalidade natural, $\mu_{h}$, e a taxa de recuperação, $\sigma$.

Os modelos considerados incluem efeitos de difusão e advecção como dependentes da densidade populacional dos mosquitos, portanto estas contribuições são não-lineares. Estas características são encontradas em vários tipos de populações de insetos [8,9]. Logo, esta modificação pode levar à melhor compreensão da influência destes efeitos na dinâmica da doença.

Inicialmente, foram estudados os dois modelos abaixo:

Modelo 1: Consideramos o modelo para a dinâmica do mosquito dado por (3), onde não é considerada a autorregulação da espécie na dinâmica homogênea, e é considerada uma difusão e advecção na parte espacial mais realista do ponto de vista biológico, que generaliza a proposta em (4). Acoplamos à esta dinâmica a população humana para estudar a disseminação da dengue. O modelo é o seguinte:

$$
\left\{\begin{aligned}
u_{t} & =\left(D(u, w) u_{x}\right)_{x}-2 \nu u^{q_{1}} u_{x}+\frac{\gamma}{k} v+\left(\frac{\gamma}{k}-\mu_{1}\right) u-\beta_{1} u i \\
w_{t} & =\left(D(u, w) w_{x}\right)_{x}-2 \nu w^{q_{2}} w_{x}+\left(\frac{\gamma}{k}-\mu_{1}\right) w+\beta_{1} u i \\
v_{t} & =k m+\left(k-\mu_{2}-\gamma\right) v \\
h_{t} & =\mu_{h} N-\mu_{h} h-\beta_{2} h w \\
i_{t} & =\beta_{2} h w-\sigma i-\mu_{h} i \\
r_{t} & =\sigma i-\mu_{h} r
\end{aligned}\right.
$$

originando um modelo que é uma continuação natural de (3), e cujos resultados poderão ser comparados aos dos artigos [3] e [4].

Modelo 2: É retirada a saturação do modelo (4) de forma a obter um modelo malthusiano, que não 
envolve a capacidade suporte $k$ :

$$
\left\{\begin{aligned}
u_{t} & =\left(D(u, w) u_{x}\right)_{x}-2 \nu u^{q_{1}} u_{x}+\gamma v-\mu_{1} u-\beta_{1} u i \\
w_{t} & =\left(D(u, w) w_{x}\right)_{x}-2 \nu w^{q_{2}} w_{x}-\mu_{1} w+\beta_{1} u i, \\
v_{t} & =m-\left(\mu_{2}+\gamma\right) v, \\
h_{t} & =\mu_{h} N-\mu_{h} h-\beta_{2} h w, \\
i_{t} & =\beta_{2} h w-\sigma I-\mu_{h} i, \\
r_{t} & =\sigma i-\mu_{h} r,
\end{aligned}\right.
$$

permitindo assim comparar resultados com um modelo já conhecido e aceito como o malthusiano.

\section{Simetrias de Lie}

Nas décadas finais do século XIX, Sophus Lie introduziu a noção de grupos contínuos de transformações, atualmente conhecidos por grupos de Lie, com o objetivo de unificar e estender vários métodos de se obter soluções de equações diferenciais, ordinárias ou parciais, que culminou com o surgimento de uma nova vertente em Matemática: a Teoria de Lie. O resultado do trabalho de Lie em equações diferenciais é o que hoje chamamos de simetrias de Lie de equações diferenciais.

Um grupo de simetrias de uma equação diferencial, ou sistema de equações, é um grupo de transformações que aplica qualquer solução da equação, ou sistema, em outra solução da mesma(o). Do ponto de vista de Lie, tal grupo depende apenas de parâmetros contínuos e consiste de transformações de pontos agindo no espaço das variáveis independentes e dependentes.

Neste trabalho, encontraremos os geradores de simetrias de Lie dos sistemas (5) e (6). Neste caso especifico, os geradores de simetrias são da forma

$$
X=\xi \frac{\partial}{\partial x}+\tau \frac{\partial}{\partial t}+\eta_{1} \frac{\partial}{\partial u}+\eta_{2} \frac{\partial}{\partial v}+\eta_{3} \frac{\partial}{\partial w}+\eta_{4} \frac{\partial}{\partial h}+\eta_{5} \frac{\partial}{\partial i}+\eta_{6} \frac{\partial}{\partial r},
$$

onde todos os coeficientes dependem de $(x, t, u, v, w, h, i, r)$. Por exemplo, mostraremos que as translações espaciais e temporais, respectivamente dadas por

$$
\begin{aligned}
(x, t, u, w, v, h, i, r) & \mapsto(x+\varepsilon, t, u, w, v, h, i, r), \\
\mathrm{e}(x, t, u, w, v, h, i, r) & \mapsto(x, t+\varepsilon, u, w, v, h, i, r),
\end{aligned}
$$

que, quando combinadas, nos levam a soluções do tipo onda viajantes. Isso nos permite definir uma nova variável $z=x+c t$, para algum parâmetro $c$, e procurar soluções para os sistemas considerados do tipo

$$
\begin{aligned}
u(x, t) & =U(z), \\
w(x, t) & =W(z), \\
& \vdots \\
r(x, t) & =R(z) .
\end{aligned}
$$

Também mostraremos outros geradores não triviais, da forma

$$
(x, t, u, w, v, h, i, r) \mapsto\left(\lambda x, t, \lambda^{p_{1}} u, \lambda^{p_{2}} w, \lambda^{p_{3}} v, \lambda^{p_{4}} h, \lambda^{p_{5}} i, \lambda^{p_{6}} r\right),
$$

para valores apropriados de $p_{1}, \ldots, p_{6}$. Maiores detalhes podem ser encontrados em Bluman \& Kumei [7] e Olver [10]. 


\section{$5 \quad$ Agradecimentos}

Felipo Bacani agradece à CAPES pelo suporte financeiro. I. L. Freire agradece à FAPESP pelo suporte financeiro através do processo 2011/19089-6 e ao CNPQ, processo n ${ }^{\circ} 308941 / 2013$ 6. M. T. agradece ao GNFM (Gruppo Nazionale per Fisica-Matematica) e à Università di Catania (através da PRA) pelo seu apoio.

\section{Referências}

[1] L. T. Takahashi, N. A. Maidana, W. C. Ferreira Jr., P. Pulino and H. M. Yang, Mathematical models for the Aedes aegypti dispersal dynamics: travelling waves by wing and wind, Bull. Math. Biol., 67 (2005) 509-528.

[2] N. A. Maidana, H. M. Yang, Describing the geographic spread of dengue disease by traveling waves, Mathematical Biosciences, 215 (2008) 64-77.

[3] I. L. Freire and M. Torrisi, Symmetry methods in mathematical modeling Aedes aegypti dispersal dynamics, Nonlin. Anal. RWA, 14 (2013) 1300-1307.

[4] I. L. Freire and M. Torrisi, Similarity solutions for systems arising from an Aedes aegypti model, Commun. Nonlin. Sci. Numer. Simulat., 19 (2014) 872-879.

[5] L. T. Takahashi, Modelos matemáticos de epidemiologia com vetores: Simulação da propagação urbana e geográfica da dengue. Tese de Doutorado, IMECC-UNICAMP, 2006.

[6] V. Cardile, M. Torrisi and R. Tracinà, On reaction-diffusion system arising in the development of Bacterial Colonies, Proceedings of 10th International Conferecen in Modern Group Analysis, 32 (2005), página 38.

[7] G. Bluman and S. Kumei, "Symmetries and diffferential equations", Springer, 1989.

[8] J. D. Murray, "Mathematical Biology", Springer, Berlin, 2002.

[9] E. E. Holmes, M. A. Lewis, J. E. Banks and R. R. Veit, Partial Differential Equations in Ecology: Spatial Interactions and Population Dynamics, Ecology, 75(1) (1994) 17-29.

[10] P. J. Olver, "Applications of Lie groups to differential equations", GMT 107, Springer, New York, 1986. 